\title{
WHEN IS THE STUDENT T-STATISTIC ASYMPTOTICALLY STANDARD NORMAL?
}

\author{
By Evarist Giné, ${ }^{1}$ Friedrich Götze ${ }^{2}$ AND David M. Mason ${ }^{3}$ \\ University of Connecticut, Universität Bielefeld \\ and University of Delaware
}

Let $X, X_{i}, i \in \mathbb{N}$, be independent, identically distributed random variables. It is shown that the Student $t$-statistic based upon the sample $\left\{X_{i}\right\}_{i=1}^{n}$ is asymptotically $N(0,1)$ if and only if $X$ is in the domain of attraction of the normal law. It is also shown that, for any $X$, if the selfnormalized sums $U_{n}:=\sum_{i=1}^{n} X_{i} /\left(\sum_{i=1}^{n} X_{i}^{2}\right)^{1 / 2}, n \in \mathbb{N}$, are stochastically bounded then they are uniformly subgaussian that is, $\sup _{n} \mathbb{E} \exp \left(\lambda U_{n}^{2}\right)<\infty$ for some $\lambda>0$.

1. Introduction. Let $X, X_{i}, i \in \mathbb{N}$, be independent, identically distributed random variables and let

$$
S_{n}=\sum_{i=1}^{n} X_{i}, \quad V_{n}^{2}=\sum_{i=1}^{n} X_{i}^{2}, \quad n \in \mathbb{N} .
$$

Using this notation we can write the classical Student $t$-statistic as

$$
T_{n}=\frac{S_{n} / V_{n}}{\sqrt{\left(n-\left(S_{n} / V_{n}\right)^{2}\right) /(n-1)}} .
$$

The main object of this article is to prove that $T_{n}$ or, equivalently, the selfnormalized sum $S_{n} / V_{n}$, is asymptotically standard normal if and only if $X$ is in the domain of attraction of the normal law, written $X \in D A N$, and $\mathbb{E} X=0$.

A basic requirement in the proof of this result is that the moments of $S_{n} / V_{n}$ converge to those of the standard normal whenever $S_{n} / V_{n}$ is asymptotically $N(0,1)$. This is a trivial consequence of our second main result, which states that, if the sequence $\left\{S_{n} / V_{n}\right\}$ is stochastically bounded, then it is uniformly subgaussian in the sense that $\sup _{n} \mathbb{E} \exp \left\{\lambda\left(S_{n} / V_{n}\right)^{2}\right\}<\infty$ for some $\lambda>0$ or, equivalently, $\sup _{n} \mathbb{E} \exp \left\{t S_{n} / V_{n}\right\} \leq 2 \exp \left\{c t^{2}\right\}$ for all $t \in \mathbb{R}$ and some $c<\infty$. To compare this with previous results, we recall that Logan, Mallows, Rice and Shepp (1973) show, among other things, that if $X$ is in the domain of attraction of an $\alpha$-stable law, $0<\alpha \leq 2$, centered if $\alpha>1$ and symmetric if $\alpha=1$, then the sequence of self-normalized sums converges in distribution to a limit which is subgaussian, and if moreover $X$ is symmetric, then the moments of $S_{n} / V_{n}$ also converge to the corresponding moments of the limit. As

Received February 1996; revised October 1996.

${ }^{1}$ Research partially supported by the SFB 343 at Bielefeld and by NSF Grant DMS-93-00725.

${ }^{2}$ Research supported by the SFB 343 at Bielefeld.

${ }^{3}$ Research partially supported by NSF Grant DMS-95-04758.

AMS 1991 subject classifications. Primary 60F 05, 62E20.

Key words and phrases. Student $t$-statistic, self-normalized sums, domains of attraction, convergence of moments. 
a consequence of our second result, we obtain that if the sequence $\left\{S_{n} / V_{n}\right\}$ is stochastically bounded then all its subsequential limits in law are (uniformly) subgaussian and there is convergence of the moment generating function of $S_{n} / V_{n}$ along each convergent (in distribution) subsequence to the moment generating function of the limit, for all $t \in \mathbb{R}$ (there is even convergence of some square exponential moments).

If $X$ is symmetric then the self-normalized sums are al ways stochastically bounded as a consequence of Khinchin's inequality. But if $X$ is not symmetric, there are even centered random variables with good moment conditions for which $S_{n} / V_{n}$ fails to be shift tight. We present examples of this type and give as well a criterion for stochastic boundedness of self-normalized sums.

The line of research leading to our results starts perhaps with Efron (1969), who studied the limiting behavior of the Student $t$-statistic and, equivalently, of self-normalized sums in some nonstandard cases. In a more strict sense, it actually begins with the conjecture of Logan, Mallows, Rice and Shepp (1973) stating that " $S_{n} / V_{n}$ is asymptotically normal if [and perhaps only if] $X$ is in the domain of attraction of the normal law" (and $X$ is centered).

The "if" part of this conjecture follows rather easily from basic principles (Raikov's theorem), as was noticed, among others, by Maller (1981), but the parenthetical "only if" part has remained open until now for the general case of not necessarily symmetric random variables. For $X$ symmetric, Griffin and Mason (1991) attribute to Roy Erickson a beautiful proof of the fact that convergence in distribution of the self-normalized sums $S_{n} / V_{n}$ to the standard normal law does indeed imply that $X \in D A N$. This result and its method of proof directly inspired ours.

As in the symmetric case [Griffin and Mason (1991)], proving that $X \in$ $D A N$ under the assumption that the self-normalized sums are asymptotically standard normal, ultimately reduces-via O'Brien's (1980) observation that $X \in D A N$ is equivalent to $\max _{i \leq n}\left|X_{i}\right| / V_{n} \rightarrow 0$ in probability-to the analysis of the terms in the development of $\mathbb{E}\left(S_{n} / V_{n}\right)^{4}$. Most of these terms vanish in the symmetric case. The main difficulty in the absence of symmetry consists in showing that the terms which are zero under symmetry are indeed asymptotically negligible in the general case. Control of these terms is achieved by means of certain estimates (Lemma 3.1) of the moments

$$
\mathbb{E}\left(X_{1}^{m_{1}} \cdots X_{r}^{m_{r}} / V_{n}^{k}\right), m_{1}+\cdots+m_{r}=k,
$$

in combination with the result on uniform subgaussianness of $S_{n} / V_{n}$ (only the consequence on boundedness of moments is used here). The key to the proof of this last theorem is another estimate (Lemma 2.1) of the same moments (1.3) which allows us to derive a sort of converse Hölder inequality for $S_{n} / V_{n}$. The (two types of) estimates of (1.3) just alluded to, which are probably of independent interest, are the main new technical tools used in the present work.

Section 2 contains the result on boundedness of exponential moments of stochastically bounded self-normalized sums and some of its consequences, as well as examples of variables for which the self-normalized sums are not shift 
tight. Section 3 is devoted to the asymptotic normality of self-normalized sums and the Student $t$-statistic which, as indicated above, is our main goal.

Letting $T_{n}$ be the Student $t$-statistic as in (1.2) based on $n$ samples from $X$, Bentkus and Götze (1994) obtained sharp bounds for $\sup _{x \in \mathbb{R}} \mid \operatorname{Pr}\left\{T_{n}<x\right\}$ $-\Phi(x) \mid$ that depend rather explicitly on the law of $X$ and tend to zero whenever $X \in D A N$. Because of our main result, remarkably, these estimates tend to zero whenever there is convergence. For other important aspects of selfnormalized sums we refer to LePage, Woodroofe and Zinn (1981), S. Csörgö (1989) and the references therein.

Finally, here are some notational conventions. Most statements of results in this article refer to the self-normalized sums $S_{n} / V_{n}$, with $S_{n}$ and $V_{n}$ as in (1.1) from a sequence of i.i.d. random variables $X_{i}$ distributed like $X$. We implicitly assume this set-up throughout and avoid describing it in every statement. We denote by $\mathscr{L}(Y)$ the probability distribution of the random variable $Y$. Limits of probability laws are always to be understood in the sense of weak convergence, that is, in the sense of limits in distribution of the variables involved. If $V_{n}(\omega)=0$ [in which case, also $S_{n}(\omega)=0$ ], with some abuse of notation we define $S_{n}(\omega) / V_{n}(\omega):=0$; that is, we adhere to the convention $0 / 0=0$.

2. Uniform subgaussianness of stochastically bounded self-normalized sums and some consequences. The proof of the main result of this section (Theorem 2.5) is ultimately based on the observation that the components in the development of $\mathbb{E}\left(S_{n} / V_{n}\right)^{k}, k>1$, are dominated, up to precise multiplicative constants, by powers of moments of order one, $\left[\mathbb{E}\left|S_{\ell} / V_{\ell}\right|\right]^{s}$, for some $\ell \leq n$ and $s \leq k$. We isolate this fact as the following lemma.

LEMMA 2.1. Let $r, k, n, m_{1}, \ldots, m_{r}$ be natural numbers such that $1 \leq r \leq$ $k \leq n, m_{i} \geq 1$ for all $i$ and $m_{1}+\cdots+m_{r}=k$. Define $n_{r}=[n / r]$ and $s=\# i \leq$ $\left.r: m_{i}=1\right\}$. Then the following inequality holds for any set of i.i.d. random variables $X_{i}, i \leq n$ :

$$
n_{r}^{r}\left(\begin{array}{c}
k \\
m_{1}, \ldots, m_{r}
\end{array}\right)^{1 / 2}\left|\mathbb{E} \frac{X_{1}^{m_{1}} \cdots X_{r}^{m_{r}}}{V_{n}^{k}}\right| \leq\left(\mathbb{E}\left|\frac{S_{n_{r}}}{V_{n_{r}}}\right|\right)^{s} .
$$

Proof. Setting

$$
S(i):=\sum_{j=1}^{n_{r}} X_{(i-1) n_{r}+j}^{m_{i}}, \quad V(i):=\left[\sum_{j=1}^{n_{r}} X_{(i-1) n_{r}+j}^{2}\right]^{1 / 2} \text { for } i=1, \ldots, r,
$$

we have

$$
n_{r}^{r}\left|\mathbb{E} \frac{X_{1}^{m_{1}} \cdots X_{r}^{m_{r}}}{V_{n}^{k}}\right|=\left|\mathbb{E} \frac{S(1) \cdots S(r)}{V_{n}^{k}}\right| .
$$


Also, by the multinomial theorem,

$$
V_{n}^{k} \geq\left(\left[\sum_{j=1}^{r}(V(j))^{2}\right]^{k}\right)^{1 / 2} \geq\left(\begin{array}{c}
k \\
m_{1}, \ldots, m_{r}
\end{array}\right)^{1 / 2} \prod_{j=1}^{r}(V(j))^{m_{j}},
$$

so that

$$
\left(\begin{array}{c}
k \\
m_{1}, \ldots, m_{r}
\end{array}\right)^{1 / 2}\left|\frac{S(1) \cdots S(r)}{V_{n}^{k}}\right| \leq \prod_{i=1}^{r}\left|\frac{S(i)}{(V(i))^{m_{i}}}\right| .
$$

[Note that, provided we adhere to our convention $0 / 0=0$, this inequality holds even if some of the $V(i)$ are zero as, in this case, both sides are zero.] Since the random variables $S(i) /(V(i))^{m_{i}}$ are independent, we obtain from the above that

$$
n_{r}^{r}\left(\begin{array}{c}
k \\
m_{1}, \ldots, m_{r}
\end{array}\right)^{1 / 2}\left|\mathbb{E} \frac{X_{1}^{m_{1}} \cdots X_{r}^{m_{r}}}{V_{n}^{k}}\right| \leq \prod_{i=1}^{r} \mathbb{E}\left|\frac{S(i)}{(V(i))^{m_{i}}}\right| .
$$

Now, if $m_{i}>1$ then $S(i) \leq(V(i))^{m_{i}}$, and therefore the last product is dominated by the product of the terms for which $m_{i}=1$,

$$
\prod_{1 \leq i \leq r: m_{i}=1} \mathbb{E}\left|\frac{S(i)}{(V(i))^{m_{i}}}\right|=\left(\mathbb{E}\left|\frac{S_{n_{r}}}{V_{n_{r}}}\right|\right)^{s},
$$

giving inequality (2.1).

Inequality (2.1) produces a kind of reverse Hölder (or hypercontractivity) inequality for the variables $\left|S_{n} / V_{n}\right|$, as follows.

LEMMA 2.2. The following inequality holds for all $n, k \in \mathbb{N}$ :

$$
\left|\mathbb{E}\left(\frac{S_{n}}{V_{n}}\right)^{k}\right| \leq c(k) \max \left[1, \max _{\ell \leq n} \mathbb{E}\left|\frac{S_{\ell}}{V_{\ell}}\right|\right]^{k},
$$

where $c(k)$ can be taken to be

$$
c(k)=\left(\frac{4 e}{3}+1\right)^{k}(k !)^{1 / 2} \leq 5^{k}(k !)^{1 / 2} .
$$

Proof. For $n \leq 4 k$ we have, by Cauchy-Schwarz,

$$
\left|S_{n} / V_{n}\right|^{k} \leq n^{k / 2} \leq 2^{k} k^{k / 2}<c(k)
$$

since $k ! \geq 2 k^{k} / e^{k}$ for all $k$ (we will make repeated use of this elementary inequality). So, we can assume $n>4 k$, in which case $1 / n_{r}<\frac{4}{3} r / n$ for all $r \leq k$. Let us set

$$
M_{n}=\max \left[1, \max _{\ell \leq n} \mathbb{E}\left|\frac{S_{\ell}}{V_{\ell}}\right|\right] .
$$


Then, inequality (2.1) gives

$$
\begin{aligned}
\left|\mathbb{E}\left(\frac{S_{n}}{V_{n}}\right)^{k}\right| & =\left|\sum_{r=1}^{k} \sum_{\substack{m_{1}+\cdots+m_{r}=k \\
m_{i} \geq 1}}\left(\begin{array}{c}
n \\
r
\end{array}\right)\left(\begin{array}{c}
k \\
m_{1}, \ldots, m_{r}
\end{array}\right) \mathbb{E} \frac{X_{1}^{m_{1}} \cdots X_{r}^{m_{r}}}{V_{n}^{k}}\right| \\
& \leq M_{n}^{k} \sum_{r=1}^{k}\left(\begin{array}{c}
n \\
r
\end{array}\right) n_{r}^{-r} \sum_{\substack{m_{1}+\cdots+m_{r}=k \\
m_{i} \geq 1}}\left(\begin{array}{c}
k \\
m_{1}, \ldots, m_{r}
\end{array}\right)^{1 / 2} \\
& \leq M_{n}^{k}(k !)^{1 / 2} \sum_{r=1}^{k}\left(\frac{4}{3}\right)^{r} \frac{r^{r}}{r !}\left(\begin{array}{c}
k-1 \\
r-1
\end{array}\right) \\
& \leq \frac{1}{2} M_{n}^{k}(k !)^{1 / 2} \sum_{r=1}^{k}\left(\frac{4 e}{3}\right)^{r}\left(\begin{array}{c}
k-1 \\
r-1
\end{array}\right) \\
& \leq \frac{2 e}{3} M_{n}^{k}(k !)^{1 / 2}\left(1+\frac{4 e}{3}\right)^{k-1}<c(k) M_{n}^{k},
\end{aligned}
$$

where we use that \# $\left.\left(m_{1}, \ldots, m_{r}\right): m_{1}+\cdots+m_{r}=k, m_{i} \geq 1, m_{i} \in \mathbb{N}\right\}=\left(\begin{array}{l}k-1 \\ r-1\end{array}\right)$ and bound the multinomial coefficients by $k$ !.

Lemma 2.2 has the following immediate consequence:

LEMMA 2.3. With $M_{n}$ defined by (2.4), we have that, for all $n \in \mathbb{N}$,

$$
\mathbb{E} \exp \left\{\frac{\left(S_{n} / V_{n}\right)^{2}}{4(1+4 e / 3)^{2} M_{n}^{2}}\right\} \leq 2
$$

and

$$
\mathbb{E} \exp \left\{t\left|S_{n} / V_{n}\right|\right\} \leq 2 \exp \left\{(1+4 e / 3)^{2} M_{n}^{2} t^{2}\right\}
$$

for all $t>0$.

Proof. Let us fix $n$ and set

$$
\lambda_{0}=\frac{1}{4(1+4 e / 3)^{2} M_{n}^{2}} .
$$

Then, inequality (2.2) and the definition of $c(k)$ readily give

$$
\begin{aligned}
\mathbb{E} \exp \left\{\lambda_{0}\left(\frac{S_{n}}{V_{n}}\right)^{2}\right\} & \leq \sum_{k=0}^{\infty} \frac{\lambda_{0}^{k}((2 k) !)^{1 / 2}(1+4 e / 3)^{2 k} M_{n}^{2 k}}{k !} \\
& <\sum_{k=0}^{\infty} \lambda_{0}^{k} 2^{k}\left(1+\frac{4 e}{3}\right)^{2 k} M_{n}^{2 k}=2
\end{aligned}
$$


since $(2 k) ! \leq\left(2^{k} k !\right)^{2}$, proving (2.5). Using the inequality $|a b| \leq a^{2} / 2+b^{2} / 2$ we have, for all $t>0$,

$$
t\left|\frac{S_{n}}{V_{n}}\right| \leq \frac{1}{2}\left(2^{1 / 2} \lambda_{0}^{1 / 2}\left|\frac{S_{n}}{V_{n}}\right|\right)^{2}+\frac{1}{2}\left(\frac{t}{\left(2 \lambda_{0}\right)^{1 / 2}}\right)^{2}=\lambda_{0}\left(\frac{S_{n}}{V_{n}}\right)^{2}+\frac{t^{2}}{4 \lambda_{0}} .
$$

Then, (2.5) gives

$$
\mathbb{E} \exp \left\{t\left|\frac{S_{n}}{V_{n}}\right|\right\} \leq 2 \exp \left\{\frac{t^{2}}{4 \lambda_{0}}\right\}
$$

that is, (2.6).

Inequality (2.2) also allows us to apply Paley-Zygmund's argument [e.g., Kahane (1968), page 6, or Kwapień and Woyczyński (1992), Lemma 0.2.1] along a subsequence and conclude that if the sequence $\left\{S_{n} / V_{n}\right\}$ is stochastically bounded then it is $L_{1}$-bounded, as follows.

LEMMA 2.4. If the sequence $\left\{S_{n} / V_{n}\right\}$ is stochastically bounded then

$$
\sup _{n} \mathbb{E}\left|\frac{S_{n}}{V_{n}}\right|<\infty
$$

Proof. Suppose, to the contrary, that

$$
\sup _{n} \mathbb{E}\left|\frac{S_{n}}{V_{n}}\right|=\infty
$$

and, for each $\ell \in \mathbb{N}$, define

$$
n_{\ell}=\min \left\{n: \mathbb{E}\left|\frac{S_{n}}{V_{n}}\right|>\ell^{1 / 2}\right\} .
$$

Then,

$$
n_{\ell}<\infty \text { for all } \ell \in \mathbb{N} \text { and } n_{\ell}>\ell \rightarrow \infty \text {. }
$$

(The number $n_{\ell}$ is finite by the assumption, and $n_{\ell}>\ell$ because, as observed above, $\left|S_{n} / V_{n}\right| \leq n^{1 / 2}$ for all $n$.) Moreover, the definition of $n_{\ell}$ implies

$$
\max _{n \leq n_{\ell}}\left|\frac{S_{n}}{V_{n}}\right|=\mathbb{E}\left|\frac{S_{n_{\ell}}}{V_{n_{\ell}}}\right|>\ell^{1 / 2}
$$

Therefore, inequality (2.2) for $n=n_{\ell}$ and $k=2$ becomes

$$
\mathbb{E}\left(\frac{S_{n_{\ell}}}{V_{n_{\ell}}}\right)^{2} \leq c(2)\left(\mathbb{E}\left|\frac{S_{n_{\ell}}}{V_{n_{\ell}}}\right|\right)^{2} .
$$

Now we observe that, by Hölder, for all $a>0$,

$$
\left.\mathbb{E}\left|\frac{S_{n}}{V_{n}}\right| \leq a+\mathbb{E}\left(\left|\frac{S_{n}}{V_{n}}\right| I\left(\left|\frac{S_{n}}{V_{n}}\right|>a\right)\right) \leq a+\left[\mathbb{E}\left(\frac{S_{n}}{V_{n}}\right)^{2}\right]^{1 / 2}\left[\operatorname{Pr}\left\{\left|\frac{S_{n}}{V_{n}}\right|>a\right)\right\}\right]^{1 / 2} .
$$


Then, this last inequality for $a=a_{\ell}=\mathbb{E}\left|S_{n_{\ell}} / V_{n_{\ell}}\right| / 2$, in combination with inequality (2.8), gives

$$
\left(\operatorname{Pr}\left\{\left|\frac{S_{n_{\ell}}}{V_{n_{\ell}}}\right|>a_{\ell}\right\}\right)^{1 / 2} \geq \frac{\frac{1}{2} \mathbb{E}\left|S_{n_{\ell}} / V_{n_{\ell}}\right|}{\left[\mathbb{E}\left(S_{n_{\ell}} / V_{n_{\ell}}\right)^{2}\right]^{1 / 2}} \geq \frac{1}{2 \sqrt{c(2)}}>0
$$

for all $\ell \in \mathbb{N}$ (this is Paley-Zygmund's argument). Since $n_{\ell}>\ell \rightarrow \infty$ and $a_{\ell}>\ell^{1 / 2} / 2 \rightarrow \infty$ as $\ell \rightarrow \infty$, it follows that the sequence $\left\{S_{n} / V_{n}\right\}_{n=1}^{\infty}$ is not stochastically bounded, a contradiction.

Combining Lemmas 2.3 and 2.4 we obtain the main result of this section.

THEOREM 2.5. Let the sequence $\left\{S_{n} / V_{n}\right\}$ be stochastically bounded. Then

$$
M:=\max \left[1, \sup _{n} \mathbb{E}\left|\frac{S_{n}}{V_{n}}\right|\right]<\infty
$$

and, moreover,

$$
\sup _{n} \mathbb{E} \exp \left\{\frac{\left(S_{n} / V_{n}\right)^{2}}{4(1+4 e / 3)^{2} M^{2}}\right\} \leq 2
$$

and

$$
\sup _{n} \mathbb{E} \exp \left\{t\left|S_{n} / V_{n}\right|\right\} \leq 2 \exp \left\{(1+4 e / 3)^{2} M^{2} t^{2}\right\}
$$

for all $t>0$.

Proof. By Lemma 2.4, $M<\infty$ and inequalities (2.10) and (2.11) follow from (2.5) and (2.6) in Lemma 2.3 since $M \geq M_{n}$ for all $n$.

A random variable $Z$, or its probability law, is subgaussian if $\operatorname{Pr}\{|Z|>t\}=$ $O\left(e^{-c t^{2}}\right)$ as $t \rightarrow \infty$, for some $c<\infty$ or, equivalently, if $\mathbb{E} e^{t Z} \leq e^{\tau t^{2}}$ for all $t \in \mathbb{R}$ and some $\tau<\infty$ [see Kahane (1968), page 62]. Theorem 2.5 then shows that if the self-normalized sums are uniformly tight, they are automatically uniformly subgaussian.

Corollary 2.6. Assume the sequence $\left\{S_{n} / V_{n}\right\}$ is stochastically bounded, let $Z$ be any of its subsequential limits in law and let $\left\{n_{k}\right\}$ be a subsequence such that

$$
\lim _{k \rightarrow \infty} \mathscr{L}\left(S_{n_{k}} / V_{n_{k}}\right)=\mathscr{L}(Z)
$$

Then $Z$ is subgaussian and

$$
\lim _{k \rightarrow \infty} \mathbb{E} \exp \left\{\frac{\left(S_{n_{k}} / V_{n_{k}}\right)^{2}}{4(1+4 e / 3)^{2} M^{2}}\right\}=\mathbb{E} \exp \left\{\frac{Z^{2}}{4(1+4 e / 3)^{2} M^{2}}\right\} \leq 2 .
$$

Hence,

$$
\lim _{k \rightarrow \infty} \mathbb{E} \exp \left\{t\left(S_{n_{k}} / V_{n_{k}}\right)\right\}=\mathbb{E} \exp \{t Z\} \leq 2 \exp \left\{(1+4 e / 3)^{2} M^{2} t^{2}\right\}
$$


for all $t \in \mathbb{R}$ and, in particular, the moments of $S_{n_{k}} / V_{n_{k}}$ also converge to the corresponding moments of $Z$.

Proof. Letting $\lambda=\left(4(1+4 e / 3)^{2} M^{2}\right)^{-1}$, which is positive by Theorem 2.5, the proof of Lemma 2.3 shows that for example, $\sup _{n} \mathbb{E} \exp \left\{(4 \lambda / 3)\left(S_{n} / V_{n}\right)^{2}\right\}<$ $\infty$, thus providing enough uniform integrability to interchange limits and expectations in the relevant sequences.

Since subgaussian variables are determined by their moments, by, for example, Proposition 8.49, page 182 in Breiman (1968), this is true in particular for any subsequential limits of a sequence of stochastically bounded selfnormalized sums. [This is relevant to the fact that, when we prove bel ow that if the self-normalized sums are asymptotically $N(0,1)$ then $X \in D A N$, the only use we make of the particular form of the limit is that the second and fourth moments of $N(0,1)$ are, respectively, 1 and 3.]

Theorem 2.5 and Corollary 2.6 are best possible except perhaps for the constants in the exponents: if $X$ is in the domain of attraction of the normal law and $X$ is centered, then $S_{n} / V_{n}$ converges in law to $N(0,1)$, as mentioned in the Introduction and proved in Section 3.

The symmetric case of Theorem 2.5 deserves special consideration.

REMARK 2.7 (The symmetric case). If $X$ is symmetric, Theorem 2.5 has a stronger version with a much simpler proof as a consequence of Khinchin's inequality [e.g., K wapień and Woyczyński (1990), inequality (3.4.3)]. Let $\left\{\varepsilon_{i}\right\}_{i=1}^{\infty}$ be a Rademacher sequence independent of the sequence $\left\{X_{i}\right\}_{i=1}^{\infty}$. (The variables $\varepsilon_{i}$ are i.i.d. and $\operatorname{Pr}\left\{\varepsilon_{i}=1\right\}=\operatorname{Pr}\left\{\varepsilon_{i}=-1\right\}=1$ 2.) Then, by symmetry, the sequences $\left\{X_{i}\right\}$ and $\left\{\varepsilon_{i} X_{i}\right\}$ have the same joint distributions. This and Khinchin's inequality readily give

$$
\begin{aligned}
\underset{n}{\sup } \mathbb{E}\left|\frac{S_{n}}{V_{n}}\right|^{k} & =\sup _{n} \mathbb{E}\left|\frac{\sum_{i=1}^{n} \varepsilon_{i} X_{i}}{V_{n}}\right|^{k} \\
& =\sup _{n} \mathbb{E} \mathbb{E}_{\varepsilon}\left|\frac{\sum_{i=1}^{n} \varepsilon_{i} X_{i}}{V_{n}}\right|^{k} \\
& \leq(k-1)^{k / 2} \sup _{n} \mathbb{E}\left[\frac{\sum_{i=1}^{n} X_{i}^{2}}{V_{n}^{2}}\right]^{k / 2} \\
& =(k-1)^{k / 2} \sup _{n} \operatorname{Pr}\left\{V_{n} \neq 0\right\} \\
& =(k-1)^{k / 2},
\end{aligned}
$$

where $\mathbb{E}_{\varepsilon}$ denotes conditional expectation given $X_{i}, i \in \mathbb{N}$. As a consequence of these inequalities, it follows that $\left\{S_{n} / V_{n}\right\}$ is al ways stochastically bounded when $X$ is symmetric. Moreover, proceeding as in the proof of Theorem 2.5 but using the estimate (2.14) instead of inequality (2.2), we obtain

$$
\sup _{n} \mathbb{E} \exp \left\{\lambda\left(S_{n} / V_{n}\right)^{2}\right\}<\infty
$$

for $\lambda<(2 e)^{-1}$, which is an improvement on inequality (2.9). 
In view of the previous remark, we may ask whether the sequence of selfnormalized sums is stochastically bounded for all random variables $X$, symmetric or not. A (related) question that one may also ask is whether such a result, as well as Theorem 2.5, follows in fact from the inequalities (2.14) by some sort of symmetrization argument. Both questions have a negative answer since, as we see immediately below, there exist centered random variables $X$ for which the sequence $\left\{S_{n} / V_{n}-c_{n}\right\}_{n=1}^{\infty}$ is not stochastically bounded for any choices of centering constants $c_{n}$ (that is, it is not shift tight) but, on the other hand, by Remark 2.6, the sequence of randomized self-normalized sums $\sum_{i=1}^{n} \varepsilon_{i} X_{i} / V_{n}, n \in \mathbb{N}$, is stochastically bounded, which is in contrast with the case of numerical normalizations.

EXAMPLE 2.8. Let $X$ be a random variable such that $\mathbb{E} X=0$ and, for some $1<\alpha<2$,

$$
\operatorname{Pr}\left\{X=2^{2^{k}}\right\}=c_{1} 2^{-\alpha 2^{k}}, \quad k=0,1,2, \ldots, \operatorname{Pr}\left\{X=-c_{2}\right\}=c_{3},
$$

with $c_{1} \neq 0$. We will show that if $\left\{X_{i}\right\}$ is a sequence of i.i.d. random variables with the law of $X$, then there is no sequence of constants $\left\{c_{n}\right\}$ such that the sequence $\left\{S_{n} / V_{n}-c_{n}\right\}$ of shifted self-normalized sums is stochastically bounded. This will be a direct consequence of the following fact: the row sums of the triangular array of two-dimensional random vectors:

$$
\mathrm{Y}_{k, i}=\left(\frac{X_{i}}{2^{2^{k}}}, \frac{X_{i}^{2}}{2^{2^{k+1}}}\right), \quad 1 \leq i \leq n_{k}:=\left[2^{\alpha 2^{k}}\right],
$$

satisfy

$$
\lim _{k \rightarrow \infty} \mathscr{L}\left(\sum_{i=1}^{n_{k}} \mathrm{Y}_{k, i}\right)=\mathscr{L}\left(N_{c_{1}}-c_{1}, N_{c_{1}}\right),
$$

where $N_{c_{1}}$ is a Poisson random variable with parameter $c_{1}$. Equation (2.15) follows in a standard way from the general central limit theorem in $\mathbb{R}^{2}$ [the $\mathrm{CLT}$ in $\mathbb{R}^{d}$ is usually stated for the Euclidean norm, but the details in the derivation of (2.15), which we omit, work better if one takes instead the norm $\left|\left(x_{1}, x_{2}\right)\right|:=\max \left(\left|x_{1}\right|,\left|x_{2}\right|\right)$; for a precise statement in any norm, see for example Theorem 7.7, Chapter 3, in Araujo and Giné (1980), taking into account that condition (3) there is automatically satisfied for finite-dimensional Banach spaces]. As a consequence of (2.15), letting $0<\tau<c_{1} \wedge 1,0<\delta<1$, we obtain

$$
\begin{aligned}
& \lim _{M \rightarrow \infty} \limsup _{n} \operatorname{Pr}\left\{\left|\frac{S_{n}}{V_{n}}\right|>M\right\} \\
& \quad \geq \lim _{\delta \rightarrow 0} \limsup \operatorname{Pr}\left\{\frac{\sum_{i=1}^{n_{k}} X_{i}}{2^{2^{k}}} \in\left(-c_{1}-\tau,-c_{1}+\tau\right), \frac{\sum_{i=1}^{n_{k}} X_{i}^{2}}{2^{2^{k+1}}}<\delta\right\} \\
& \quad=\lim _{\delta \rightarrow 0} \operatorname{Pr}\left\{N_{c_{1}} \in(-\tau, \tau), N_{c_{1}}<\delta\right\} \\
& \quad=\lim _{\delta \rightarrow 0} \operatorname{Pr}\left\{N_{c_{1}}=0\right\}=e^{-c_{1}}>0,
\end{aligned}
$$


showing that the sequence $\left\{S_{n} / V_{n}\right\}$ is not stochastically bounded. Finally, we show that this sequence is not shift tight either; that is, that there is no sequence of numerical constants $c_{n}$ for which the shifted sequence $\left\{\left(S_{n} / V_{n}\right)-\right.$ $\left.c_{n}\right\}$ is stochastically bounded. By the previous computation, such a sequence necessarily satisfies that $\left|c_{n_{b}}\right| \rightarrow \infty$. On the other hand, if $r \in \mathbb{N}$ is such that $r>c_{1}+1$ and $0<\tau<1$, and we set $J:=\left(\left(r-\tau-c_{1}\right) / \sqrt{r+\tau},\left(r+\tau-c_{1}\right) / \sqrt{r-\tau}\right)$ then, obviously, $\left|c_{n_{k}}\right| \rightarrow \infty$ implies that, for all $M>0$, the shifted interval $J-c_{n_{k}}$ is eventually contained in $\{|x|>M\}$, and therefore we have, again by (2.15),

$$
\begin{aligned}
& \underset{k}{\liminf } \operatorname{Pr}\left\{\left|\frac{S_{n_{k}}}{V_{n_{k}}}-c_{n_{k}}\right|>M\right\} \\
& \quad \geq \liminf _{k} \operatorname{Pr}\left\{\frac{S_{n_{k}}}{V_{n_{k}}} \in J\right\} \\
& \geq \lim _{k} \operatorname{Pr}\left\{r-\tau-c_{1}<S_{n_{k}} / 2^{2^{k}}<r+\tau-c_{1}, r-\tau<V_{n_{k}}^{2} / 2^{2^{k+1}}<r+\tau\right\} \\
& \quad=\operatorname{Pr}\left\{N_{c_{1}}=r\right\}>0,
\end{aligned}
$$

contradicting the stochastic boundedness of the sequence $\left\{\left(S_{n_{k}} / V_{n_{k}}\right)-c_{n}\right\}$.

Example 2.8 shows that for every $1<\tau<2$ there are centered random variables $X$ with finite absolute moment of order $\tau$ for which the sequence $\left\{S_{n} / V_{n}\right\}$ is not shift tight.

EXAMPLE 2.9. Let $X$ be a random variable in the domain of attraction of a stable law of index $\alpha=1$, written $X \in D(1)$. It is easy to see from the general central limit theorem in $\mathbb{R}^{2}$ and from the properties of $D(1)$ that there exist constants $a_{n} \nearrow \infty$ and $b_{n}$ such that

$$
\lim _{n \rightarrow \infty} \mathscr{L}\left(\frac{S_{n}-b_{n}}{a_{n}}, \frac{V_{n}^{2}}{a_{n}^{2}}\right)=\mathscr{L}\left(\theta_{1}, \theta_{1 / 2}\right),
$$

where $\theta_{1}$ is 1-stable and $\theta_{1 / 2}$ is positive and $\frac{1}{2}$-stable [see Csörgö, Haeusler and Mason (1988), and Csörgö (1989), for an approach to domains of attraction based on quantiles and Feller (1971), Section XVII.5, or Araujo and Giné (1980), Section 2.6, for the classical approach]. Clearly then, whenever $\left|b_{n} / a_{n}\right| \rightarrow \infty$ it is impossible for $\left\{S_{n} / V_{n}\right\}_{n=1}^{\infty}$ to be shift tight. The following example shows that this can even happen when $\mathbb{E} X=0$. Let $X$ be any centered random variable whose tail probabilities are of the order

$$
\operatorname{Pr}\{X>t\} \sim \frac{c_{1}}{t(\log t)(\log \log t)^{2}}, \quad \operatorname{Pr}\{X<-t\} \sim \frac{c_{2}}{t(\log t)(\log \log t)^{2}}
$$

as $t \rightarrow \infty$, with $c_{1} \neq c_{2}$ and at least one of the constants $c_{1}$ and $c_{2}$ different from zero. Concretely we can take $X$ to be such that its quantile function, defined as $Q(s)=\inf \{x: \operatorname{Pr}\{X \leq x\} \geq s\}, 0<s<1$, is precisely

$$
Q(s)=-h(s)+\int_{0}^{1} h(s) d s
$$


with $h(s)=s^{-1}\left(L\left(s^{-1}\right)\right)^{-1}\left(L L\left(s^{-1}\right)\right)^{-2}$ and $L(s):=\log (s \vee e)$. Then, by choosing $a_{n}$ and $b_{n}$ as in Corollary 3 of Csörgö, Haeusler and Mason (1988), it is readily checked that

$$
b_{n} \sim \frac{-n}{\log n \log \log n} \quad \text { and } \quad a_{n} \sim \frac{n}{\log n(\log \log n)^{2}},
$$

so that $\left|b_{n} / a_{n}\right| \rightarrow \infty$.

This example is interesting in that it shows that $\left\{S_{n} / V_{n}\right\}$ may fail to be shift tight even if $X$ is centered and there are numerical normalizers $a_{n}$ for which the sequence $\left\{S_{n} / a_{n}\right\}$ is shift tight.

In view of the previous examples, it makes sense to ask for analytic conditions ensuring stochastic boundedness of self-normalized sums. Although this subject is tangential to the main object of this article, we present one such necessary and sufficient condition as another corollary of Theorem 2.5.

Corollary 2.10. A necessary and sufficient condition for the sequence of self-normalized sums $\left\{S_{n} / V_{n}\right\}_{n=1}^{\infty}$ to be stochastically bounded is that for some $\delta>0$,

$$
\underset{n}{\limsup } n^{2} \int_{0}^{\delta}\left[\mathbb{E} X \exp \left(-\lambda X^{2}\right)\right]^{2}\left[\mathbb{E} \exp \left(-\lambda X^{2}\right)\right]^{n-2} d \lambda<\infty .
$$

Proof. If $X$ is degenerate, the result is trivial, so we can assume $X$ takes more than one value. By Corollary 2.6 the sequence $\left\{S_{n} / V_{n}\right\}_{n=1}^{\infty}$ is stochastically bounded if and only if the sequence of second moments is bounded. Since $\mathbb{E}\left(S_{n} / V_{n}\right)^{2}=1+\mathbb{E}\left(\sum_{1 \leq i \neq j \leq n} X_{i} X_{j} / V_{n}^{2}\right)$, stochastic boundedness of the self-normalized sums is equivalent to the condition

$$
\limsup _{n \rightarrow \infty} n^{2} \mathbb{E} \frac{X_{1} X_{2}}{V_{n}^{2}}<\infty .
$$

For any $\delta>0$ we can write

$$
\frac{X_{1} X_{2}}{V_{n}^{2}}=\int_{0}^{\delta} X_{1} X_{2} \exp \left(-\lambda V_{n}^{2}\right) d \lambda+\int_{\delta}^{\infty} X_{1} X_{2} \exp \left(-\lambda V_{n}^{2}\right) d \lambda
$$

(this identity is obvious for $V_{n} \neq 0$, and it holds also for $V_{n}=0$ because of the convention in force for 0/0.) Now, since $\left|\sum_{1 \leq i \neq j \leq n} X_{i} X_{j}\right| \leq \sum_{i, j \leq n}\left(X_{i}^{2}+\right.$ $\left.X_{j}^{2}\right) / 2-V_{n}^{2}=(n-1) V_{n}^{2}$, we have

$$
\begin{aligned}
\mathbb{E} \int_{\delta}^{\infty} X_{1} X_{2} \exp \left(-\lambda V_{n}^{2}\right) d \lambda & =\mathbb{E}\left[\frac{X_{1} X_{2}}{V_{n}^{2}} \exp \left(-\delta V_{n}^{2}\right)\right] \\
& =\frac{1}{n(n-1)} \mathbb{E}\left[\sum_{1 \leq i \neq j \leq n} \frac{X_{i} X_{j}}{V_{n}^{2}} \exp \left(-\delta V_{n}^{2}\right)\right] \\
& \leq \frac{1}{n} \mathbb{E} \exp \left(-\delta V_{n}^{2}\right) \\
& =\frac{1}{n}\left[\mathbb{E} \exp \left(-\delta X^{2}\right)\right]^{n} .
\end{aligned}
$$


With $X$ being nondegenerate, there exists $\tau>0$ such that $\operatorname{Pr}\{|X| \leq \tau\}<1$ and therefore,

$$
\mathbb{E} \exp \left(-\frac{\delta}{2} X^{2}\right) \leq \operatorname{Pr}\{|X| \leq \tau\}+\exp \left(-\frac{\delta \tau^{2}}{2}\right) \operatorname{Pr}\{|X|>\tau\}:=\sigma_{\delta}<1 .
$$

Hence,

$$
n^{2} \mathbb{E} \int_{\delta}^{\infty} X_{1} X_{2} \exp \left(-\lambda V_{n}^{2}\right) d \lambda \leq n \sigma_{\delta}^{n} \rightarrow 0 \quad \text { as } n \rightarrow \infty
$$

for all $\delta>0$. Since

$$
\mathbb{E} \int_{0}^{\delta} X_{1} X_{2} \exp \left(-\lambda V_{n}^{2}\right) d \lambda=\int_{0}^{\delta}\left[\mathbb{E} X \exp \left(-\lambda X^{2}\right)\right]^{2}\left[\mathbb{E} \exp \left(-\lambda X^{2}\right)\right]^{n-2} d \lambda,
$$

the proposition follows from (2.18) and (2.19).

In connection with (2.16), it is interesting to note that if $\mathbb{E} X=0$ then $\mathbb{E}\left[X \exp \left(-\lambda X^{2}\right)\right]=-\lambda \mathbb{E}\left[X^{3} \exp \left(-\lambda \theta X^{2}\right)\right]$, where $\theta$ is uniform on $[0,1]$, independent of $X$. For example, using Corollary 2.10 and this observation it can be proved without much effort that if $X$ is centered and there exist $C>0$ and $\alpha>1$ such that $\limsup _{n \rightarrow \infty} \mathbb{E} X^{2} I(|X| \leq t x) / \mathbb{E} X^{2} I(|X| \leq t) \leq C x^{2-\alpha}$ for all $x>1$, then the sequence $\left\{S_{n} / V_{n}\right\}$ is stochastically bounded [this condition means that $X$ is in the Feller class, "above $\alpha=1$ "; see Feller (1966), Section 9].

3. The Student t-statistic and the domain of attraction of the normal law. If we only assume stochastic boundedness of the self-normalized sums, then Lemma 2.1 and Theorem 2.5 give that $\mathbb{E}\left(X_{1}^{m_{1}} \cdots X_{r}^{m_{r}} / V_{n}^{k}\right)=$ $O\left(n^{-r}\right)$, a bound that cannot in general be improved. However, the proof of our main theorem requires improving this bound to $o\left(n^{-r}\right)$ when $m_{i}=1$ for at least one $1 \leq i \leq r$, under the assumption that the self-normalized sums are asymptotically $N(0,1)$. This is what the following Hölder type inequality will do for us.

LEMMA 3.1. Let $r, k, n, m_{1}, \ldots, m_{r}$ be natural numbers such that $k$ is even, $1 \leq r \leq k, m_{i} \geq 1$ for all $i, m_{1}+\cdots+m_{r}=k$ and $n \geq \max \left(2 m_{1}, 2(k-\right.$ $\left.m_{1}\right)$ ). Then, the following inequality holds for any set of i.i.d. random variables $X_{i}, i \leq n$ :

$$
\begin{aligned}
\left|\mathbb{E} \frac{X_{1}^{m_{1}} \cdots X_{r}^{m_{r}}}{V_{n}^{k}}\right| \leq & \frac{\left[\left(m_{1}-1\right) !\left(k-m_{1}-1\right) !\right]^{1 / 2}}{((k / 2)-1) !}\left[\mathbb{E} \frac{X_{1}^{m_{1}} X_{2}^{m_{1}}}{V_{n}^{2 m_{1}}}\right]^{1 / 2} \\
& \times\left[\mathbb{E} \frac{X_{3}^{m_{2}} X_{4}^{m_{2}} \cdots X_{2 r-1}^{m_{r}} X_{2 r}^{m_{r}}}{V_{n}^{2\left(k-m_{1}\right)}}\right]^{1 / 2},
\end{aligned}
$$

where the expected values at the right-hand side are nonnegative. 
PROOF. We apply the el ementary identity

$$
\int_{0}^{\infty} \lambda^{m-1} \exp (-\lambda a) d \lambda=\frac{(m-1) !}{a^{m}},
$$

which is valid for all positive integers $m$ and positive real numbers $a$, with $a=V_{n}^{2}$ and $m=k / 2$, to obtain

$$
\mathbb{E} \frac{X_{1}^{m_{1}} \cdots X_{r}^{m_{r}}}{V_{n}^{k}}=\frac{1}{((k / 2)-1) !} \mathbb{E} \int_{0}^{\infty} \lambda^{(k / 2)-1} X_{1}^{m_{1}} \cdots X_{r}^{m_{r}} \exp \left(-\lambda V_{n}^{2}\right) d \lambda .
$$

[Because of the $0 / 0=0$ convention, this identity is true even if $V_{n}(\omega)$ is not different from zero for all $\omega$.] The order of the integrals can be reversed since $\left(\left|X_{1}^{m_{1}} \cdots X_{r}^{m_{r}}\right| / V_{n}^{k}\right) I\left(V_{n} \neq 0\right) \leq 1$. Hence, setting

$$
\varphi(\lambda)=\mathbb{E} \exp \left(-\lambda X^{2}\right)
$$

we have

$$
\begin{aligned}
\mathbb{E} \frac{X_{1}^{m_{1}} \cdots X_{r}^{m_{r}}}{V_{n}^{k}}= & \frac{1}{((k / 2)-1) !} \int_{0}^{\infty} \lambda^{(k / 2)-1} \mathbb{E}\left[X_{1}^{m_{1}} \cdots X_{r}^{m_{r}} \exp \left(-\lambda V_{n}^{2}\right)\right] d \lambda \\
= & \frac{1}{((k / 2)-1) !} \int_{0}^{\infty} \lambda^{(k / 2)-1}\left[\prod_{j=1}^{r}\left(\mathbb{E} X^{m_{j}} \exp \left(-\lambda X^{2}\right)\right)\right] \\
& \times\left[\mathbb{E} \exp \left(-\lambda X^{2}\right)\right]^{n-r} d \lambda \\
= & \frac{1}{((k / 2)-1) !} \int_{0}^{\infty}\left[\prod_{j=1}^{r} \frac{\lambda^{m_{j} / 2} \mathbb{E} X^{m_{j}} \exp \left(-\lambda X^{2}\right)}{\varphi(\lambda)}\right] \frac{\varphi^{n}(\lambda)}{\lambda} d \lambda .
\end{aligned}
$$

Now we can apply Hölder's inequality with respect to the measure $d \mu(\lambda)=$ $\lambda^{-1} \varphi^{n}(\lambda) d \lambda$, and obtain from (3.2) that

$$
\begin{aligned}
\left|\mathbb{E} \frac{X_{1}^{m_{1}} \cdots X_{r}^{m_{r}}}{V_{n}^{k}}\right| \leq & \frac{1}{((k / 2)-1) !}\left[\int_{0}^{\infty}\left(\frac{\lambda^{m_{1} / 2} \mathbb{E} X^{m_{1}} \exp \left(-\lambda X^{2}\right)}{\varphi(\lambda)}\right)^{2} \frac{\varphi^{n}(\lambda)}{\lambda} d \lambda\right]^{1 / 2} \\
& \times\left[\int_{0}^{\infty}\left(\prod_{j=2}^{r} \frac{\lambda^{m_{j} / 2} \mathbb{E} X^{m_{j}} \exp \left(-\lambda X^{2}\right)}{\varphi(\lambda)}\right)^{2} \frac{\varphi^{n}(\lambda)}{\lambda} d \lambda\right]^{1 / 2} \\
= & \frac{\left[\left(m_{1}-1\right) !\left(k-m_{1}-1\right) !\right]^{1 / 2}}{((k / 2)-1) !}\left[\mathbb{E} \frac{X_{1}^{m_{1}} X_{2}^{m_{1}}}{V_{n}^{2 m_{1}}}\right]^{1 / 2} \\
& \times\left[\mathbb{E} \frac{X_{3}^{m_{2}} X_{4}^{m_{2}} \cdots X_{2 r-1}^{m_{r}} X_{2 r}^{m_{r}}}{V_{n}^{2\left(k-m_{1}\right)}}\right]^{1 / 2},
\end{aligned}
$$

and it is clear from these identities that the last two expected values are nonnegative.

Proving that if $X \in D A N$ and $\mathbb{E} X=0$ then the self-normalized sums are asymptotically normal is a simple consequence of Raikov's theorem as observed, for example, by Maller (1981). For this, as well as for proving that 
$X$ is centered if $S_{n} / V_{n}$ is asymptotically normal, we require a small variation on Raikov's theorem, and we include its very simple proof for the reader's convenience.

LEMMA 3.2. Let $X, X_{i}, i \in \mathbb{N}$, be i.i.d. random variables in the domain of attraction of the normal law and such that $\mathbb{E} X^{2}=\infty$. Let $b_{n} \nearrow \infty$ be a set of constants for which

$$
\mathscr{L}\left(\frac{1}{b_{n}} \sum_{i=1}^{n}\left(X_{i}-\mathbb{E} X\right)\right) \rightarrow_{w} N(0,1) .
$$

Then,

$$
\frac{1}{b_{n}^{2}} \sum_{i=1}^{n} X_{i}^{2} \rightarrow 1 \text { in probability. }
$$

Proof. We should recall that if $X \in D A N$ then $\mathbb{E}|X|^{2-\tau}<\infty$ for all $0<$ $\tau<2$ and that constants $b_{n}$, for which (3.3) holds, always exist and have the form $b_{n}=n^{1 / 2} \ell(n)$ for some function $\ell$ slowly varying at infinity. If $\mathbb{E} X^{2}=$ $\infty$, truncated variances can be replaced by truncated second moments in the general criterion for normal convergence and therefore, if (3.3) holds, then

$$
\lim _{n \rightarrow \infty} \frac{n}{b_{n}^{2}} \mathbb{E} X^{2} I\left(|X| \leq \delta b_{n}\right)=1 \text { and } \lim _{n \rightarrow \infty} n \operatorname{Pr}\left\{|X|>\delta b_{n}\right\}=0
$$

for all $\delta>0$. [For these and other facts on domains of attraction, see Csörgő, Haeusler and Mason (1988), Feller (1971), or Araujo and Giné (1980)]. The last two limits imply that for all $\varepsilon>0$ and $\delta>0$ there is an $N<\infty$ such that, for $n \geq N$,

$$
\begin{aligned}
& \operatorname{Pr}\left\{\left|\frac{\sum_{i=1}^{n} X_{i}^{2}}{b_{n}^{2}}-1\right|>\varepsilon\right\} \\
& \leq n \operatorname{Pr}\left\{|X|>\delta b_{n}\right\} \\
& \quad+\operatorname{Pr}\left\{\left|\sum_{i=1}^{n} X_{i}^{2} I\left(\left|X_{i}\right| \leq \delta b_{n}\right)-n \mathbb{E} X^{2} I\left(\left|X_{i}\right| \leq \delta b_{n}\right)\right|>\frac{b_{n}^{2} \varepsilon}{2}\right\} \\
& \leq n \operatorname{Pr}\left\{|X|>\delta b_{n}\right\}+\frac{4 n}{\varepsilon^{2} b_{n}^{4}} \mathbb{E} X^{4} I\left(\left|X_{i}\right| \leq \delta b_{n}\right) \\
& \leq n \operatorname{Pr}\left\{|X|>\delta b_{n}\right\}+\frac{4 \delta^{2}}{\varepsilon^{2}} \frac{n}{b_{n}^{2}} \mathbb{E} X^{2} I\left(\left|X_{i}\right| \leq \delta b_{n}\right),
\end{aligned}
$$

and (3.4) follows by letting first $n \rightarrow \infty$ and then $\delta \rightarrow 0$.

We are now ready to prove our main result characterizing asymptotic normality of self-normalized sums. 
THEOREM 3.3. The following two statements are equival ent:

(a) $X$ is in the domain of attraction of the normal law and $E X=0$;

$$
\lim _{n \rightarrow \infty} \mathscr{L}\left(\frac{S_{n}}{V_{n}}\right)=N(0,1) .
$$

Proof. Assume condition (a) holds. Then, if $\mathbb{E} X^{2}<\infty$, the self-normalized sums converge in distribution to the standard normal law by the central limit theorem and the law of large numbers. If $\mathbb{E} X^{2}=\infty$, then (3.5) is a direct consequence of (3.3) and (3.4) in Lemma 3.2. So (b) is proved.

If $X \in D A N$, then the sequence $\left\{\left(S_{n}-n \mathbb{E} X\right) / V_{n}\right\}_{n=1}^{\infty}$ converges in law (as above, by the CLT and the LLN if $\mathbb{E} X^{2}<\infty$, and by Lemma 3.2 if $\mathbb{E} X^{2}=\infty$ ). Suppose for the moment that the sequence $\left\{S_{n} / V_{n}\right\}_{n=1}^{\infty}$ also converges in law. Then the sequence of centerings $n(\mathbb{E} X) / V_{n}, n \in \mathbb{N}$, must be stochastically bounded. But if $b_{n}$ is as in Lemma 3.2 for $\mathbb{E} X^{2}=\infty$, and $b_{n}=n^{1 / 2}$ for $\mathbb{E} X^{2}<\infty$, then $b_{n} / V_{n}$ converges in probability to a finite number whereas $n / b_{n} \rightarrow \infty$ [recall $b_{n}=n^{1 / 2} \ell(n)$ with $\ell(x)$ slowly varying at infinity]. Hence, we must have $\mathbb{E} X=0$.

The proof of the theorem is thus reduced to showing that if (b) holds, then $X \in D A N$. One of the several necessary and sufficient conditions for $X \in$ $D A N$ is that

$$
\lim _{n \rightarrow \infty} \frac{\max _{i \leq n}\left|X_{i}\right|}{V_{n}}=0 \text { in probability, }
$$

[O'Brien (1980)], as mentioned above. Then, since

$$
\operatorname{Pr}\left\{\max _{i \leq n} \frac{\left|X_{i}\right|}{V_{n}}>\varepsilon\right\} \leq n \operatorname{Pr}\left\{\frac{\left|X_{1}\right|}{V_{n}}>\varepsilon\right\} \leq \frac{n}{\varepsilon^{4}} \mathbb{E}\left(\frac{X_{1}}{V_{n}}\right)^{4},
$$

it suffices to prove

$$
\mathbb{E}\left(\frac{X_{1}}{V_{n}}\right)^{4}=o\left(n^{-1}\right)
$$

under the assumption that $S_{n} / V_{n}$ is asymptotically $N(0,1)$. Let us then assume (3.5). The left-hand side of (3.7) is one of the summands in the development of $\mathbb{E}\left(S_{n} / V_{n}\right)^{4}, n \geq 4$,

$$
\begin{aligned}
\mathbb{E}\left(\frac{S_{n}}{V_{n}}\right)^{4}= & \sum_{r=1}^{4} \sum_{\substack{m_{1}+\cdots+m_{r}=4 \\
m_{i} \geq 1}}\left(\begin{array}{l}
n \\
r
\end{array}\right)\left(\begin{array}{c}
4 \\
m_{1}, \ldots, m_{r}
\end{array}\right) \mathbb{E} \frac{X_{1}^{m_{1}} \cdots X_{r}^{m_{r}}}{V_{n}^{4}} \\
= & n \mathbb{E}\left(\frac{X_{1}}{V_{n}}\right)^{4}+6\left(\begin{array}{l}
n \\
2
\end{array}\right) \mathbb{E} \frac{X_{1}^{2} X_{2}^{2}}{V_{n}^{4}}+8\left(\begin{array}{l}
n \\
2
\end{array}\right) \mathbb{E} \frac{X_{1} X_{2}^{3}}{V_{n}^{4}} \\
& +36\left(\begin{array}{c}
n \\
3
\end{array}\right) \mathbb{E} \frac{X_{1} X_{2} X_{3}^{2}}{V_{n}^{4}}+24\left(\begin{array}{c}
n \\
4
\end{array}\right) \mathbb{E} \frac{X_{1} \cdots X_{4}}{V_{n}^{4}} .
\end{aligned}
$$


We will obtain (3.7) by taking limits in this identity, using the fact that the moments of $S_{n} / V_{n}$ converge, together with Lemmas 2.1 and 3.1. Since $\sum_{1 \leq i \neq j \leq n} X_{i}^{2} X_{j}^{2} / V_{n}^{4}=1-\sum_{i=1}^{n} X_{i}^{4} / V_{n}^{4}$, we have

$$
2\left(\begin{array}{l}
n \\
2
\end{array}\right) \mathbb{E} \frac{X_{1}^{2} X_{2}^{2}}{V_{n}^{4}}=1-n \mathbb{E}\left(\frac{X_{1}}{V_{n}}\right)^{4}
$$

and the previous development simplifies to

$$
\begin{aligned}
\mathbb{E}\left(S_{n} / V_{n}\right)^{4}= & 3-2 n \mathbb{E}\left(\frac{X_{1}}{V_{n}}\right)^{4}+8\left(\begin{array}{l}
n \\
2
\end{array}\right) \mathbb{E} \frac{X_{1} X_{2}^{3}}{V_{n}^{4}} \\
& +36\left(\begin{array}{l}
n \\
3
\end{array}\right) \mathbb{E} \frac{X_{1} X_{2} X_{3}^{2}}{V_{n}^{4}}+24\left(\begin{array}{l}
n \\
4
\end{array}\right) \mathbb{E} \frac{X_{1} \cdots X_{4}}{V_{n}^{4}} .
\end{aligned}
$$

Now, by Corollary 2.6, there is also convergence of moments in the limit (3.5). Hence,

$$
\lim _{n \rightarrow \infty} \mathbb{E}\left(S_{n} / V_{n}\right)^{4}=3,
$$

and therefore (3.7) will be proved if we show that the last three summands at the right of equation (3.8) tend to zero. So, we must obtain the bound

$$
\left|\mathbb{E} \frac{X_{1} X_{2}^{m_{2}} \cdots X_{r}^{m_{r}}}{V_{n}^{4}}\right|=o\left(n^{-r}\right)
$$

for all $1 \leq r \leq 4$ and $1+m_{2}+\cdots+m_{r}=4$. For this we use Lemma 3.1, which gives that, for $n \geq 6$,

$$
\left|\mathbb{E} \frac{X_{1} X_{2}^{m_{2}} \cdots X_{r}^{m_{r}}}{V_{n}^{4}}\right| \leq 2^{1 / 2}\left[\mathbb{E} \frac{X_{1} X_{2}}{V_{n}^{2}}\right]^{1 / 2}\left[\mathbb{E} \frac{X_{3}^{m_{2}} X_{4}^{m_{2}} \cdots X_{2 r-1}^{m_{r}} X_{2 r}^{m_{r}}}{V_{n}^{6}}\right]^{1 / 2} .
$$

To bound the first factor at the right of (3.11), we observe that by Corollary 2.6 and the limit (3.5),

$$
\lim _{n \rightarrow \infty} \mathbb{E}\left(S_{n} / V_{n}\right)^{2}=1
$$

which, since $\left(S_{n} / V_{n}\right)^{2}=1-\sum_{1 \leq i \neq j \leq n} X_{i} X_{j} / V_{n}^{2}$, implies

$$
\mathbb{E} \frac{X_{1} X_{2}}{V_{n}^{2}}=o\left(n^{-2}\right) \text {. }
$$

Lemma 2.4 implies, by (3.5), that $\sup _{n} \mathbb{E}\left|S_{n} / V_{n}\right|<\infty$, and this, combined with Lemma 2.1, yields

$$
\mathbb{E} \frac{X_{3}^{m_{2}} X_{4}^{m_{2}} \cdots X_{2 r-1}^{m_{r}} X_{2 r}^{m_{r}}}{V_{n}^{6}}=O\left(n^{-2(r-1)}\right) .
$$

Equations (3.11)-(3.13) give (3.10), proving the theorem. 
As mentioned in the Introduction, the classical Student $t_{n}$-statistic,

$$
T_{n}(X)=\frac{\left(1 / n^{1 / 2}\right) \sum_{i=1}^{n} X_{i}}{\left[(1 / n-1) \sum_{i=1}^{n}\left(X_{i}-\bar{X}_{n}\right)^{2}\right]^{1 / 2}},
$$

where $\bar{X}_{n}:=S_{n} / n$, satisfies equation (1.2). Therefore, if $T_{n}(X)$ or $S_{n} / V_{n}$ has a limiting distribution, so does the other and both coincide (this is well known). Hence, replacing $X$ by $X-a$ in this observation and in Theorem 3.3 (note that $X \in D A N$ iff $X-a \in D A N$ ), we obtain the theorem.

THEOREM 3.4. The fol lowing two statements are equival ent:

(i) $X$ is in the domain of attraction of the normal law;

(ii) There exists a finite constant $a$ such that

$$
\lim _{n \rightarrow \infty} \mathscr{L}\left(T_{n}(X-a)\right)=N(0,1)
$$

Moreover, if either (i) or (ii) holds, then $a=\mathbb{E} X$.

Theorem 3.4 provides a full answer to this artide's title question.

\section{REFERENCES}

Araujo, A. and Giné, E. (1980). The Central Limit Theorem for Real and Banach Valued Random Variables. Wiley, New York.

Bentkus, V. and Götze, F. (1994). The Berry-Esseen bound for Student's statistic. Ann. Probab. 24 491-503.

Breiman, L. (1968). Probability. Addison-Wesley, Reading, MA.

CsöRGÖ, S. (1989). Notes on extreme and self-normalised sums from the domain of attraction of a stable law. J. London Math. Soc. 39 369-384.

CsÖRGŐ, S., HAEUSleR, E. and MASON, D. M. (1988). A probabilistic approach to the asymptotic distribution of sums of independent, identically distributed random variables. Adv. in Appl. Math. 9 259-333.

EFron, B. (1969). Student's $t$-test under symmetry conditions. J . Amer. Statist. Assoc. $641278-$ 1302.

FelleR, W. (1966). On regular variation and local limit theorems. In Proc. Fifth Berkeley Symp. Math. Statist. Probab. 2 373-388. Univ. California Press, Berkeley.

FELleR, W. (1971). An Introduction to Probability Theory and Its Applications 2. Wiley, New York.

GRIFFIn, P. S. and MASON, D. M. (1991). On the asymptotic normality of self-normalized sums. Proc. Cambridge Phil. Soc. 109 597-610.

KAHANE, J.-P. (1968). Some Random Series of Functions. Heath, Lexington, MA.

KWAPIEŃ, S. and WoyCzyŃsKI, W. (1992). Random Series and Stochastic Integrals. Birkhäuser, Boston.

LePage, R., Woodroofe, M. and Zinn, J. (1981). Convergence to a stable distribution via order statistics. Ann. Probab. 9 624-632.

Logan, B. F., Mallows, C. L., Rice, S. O. and ShePp, L. A. (1973). Limit distributions of selfnormalized sums. Ann. Probab. 1 788-809. 
MALLER, R. A. (1981). A theorem on products of random variables, with application to regression. Austral. J. Statist. 23 177-185.

O'BRIEN, G. L. (1980). A limit theorem for sample maxima and heavy branches in Galton-Watson trees. J. Appl. Probab. 17 539-545.

E. GINÉ

DEPARTMENTS OF MATHEMATICS AND Statistics

UNIVERSITY OF CONNECTICUT

STORRS, CONNECTICUT 06269

E-MAIL: gine@uconnvm.uconn.edu
F. GÖTZE

FAKULTäT FÜR MATHEMATIK

UNIVERSITÄT BIELEFELD

POSTFACH 8640

4800 BIELEFELD 1

GERMANY

E-MAIL: goetze@mathematik.uni-bielefeld.de

D. MASON

Department of Mathematical Sciences

UNIVERSITY OF DELAWARE

NEWARK, DELAWARE 19716

E-MAIL: davidm@math.udel.edu 